\title{
Red blood cell distribution width is useful in discriminating adult onset Still's disease and sepsis within 24 hours after hospitalization
}

\author{
Hee-Jin Park ${ }^{1}$, Jungsik Song ${ }^{2}$, Yong-Beom Park ${ }^{2}$, Soo-Kon Lee ${ }^{2}$, and Sang-Won Lee ${ }^{2}$
}

${ }^{1}$ Division of Rheumatology, Department of Internal Medicine, International St. Mary's Hospital, Catholic Kwandong University College of Medicine, Incheon; ${ }^{2}$ Division of Rheumatology, Department of Internal Medicine, Yonsei University College of Medicine, Seoul, Korea

Received: March 10, 2016 Revised : August 23, 2016 Accepted: November 11, 2016

\section{Correspondence to}

Sang-Won Lee, M.D.

Division of Rheumatology,

Department of Internal

Medicine, Yonsei University

College of Medicine, 50-1

Yonsei-ro, Seodaemun-gu, Seoul 03722, Korea

Tel: +82-2-2228-1984

Fax: +82-2-363-6884

E-mail: sangwonlee@yuhs.ac
Background/Aims: Red blood cell distribution width (RDW) is a value representing the heterogeneity in the size of red blood cell, and it is usually used in distinguishing types of anaemia. Recently, it was reported that it could reflect the burden of inflammation in diverse diseases and their prognosis. Hence, in this study, we investigated whether RDW may contribute to discriminating adult onset Still's disease (AOSD) from sepsis in serious febrile patients within 24 hours after hospitalization.

Methods: We reviewed the medical records and enrolled 21 AOSD patients, 27 sepsis patients and 30 matched healthy controls. We collected at least two laboratory results of variables including RDW within 24 hours after hospitalization, and we calculated their mean values.

Results: Sepsis patients showed the significantly increased median white blood cell count, compared to AOSD patients $\left(14,390.0 / \mathrm{mm}^{3} \mathrm{vs} .12,390.0 / \mathrm{mm}^{3}, p=0.010\right)$. The median RDW in sepsis patients was higher than that in AOSD patients $(15.0 \%$ vs. $13.3 \%, p=0.001$ ), and furthermore, the median RDW in both patient-groups was significantly higher than that in healthy controls. In contrast, the median ferritin level in sepsis patients was lower than that in AOSD patients (544.0 mg/dL vs. 3,756.6 $\mathrm{mg} / \mathrm{dL}, p=0.001$ ). In multivariate analysis, $\mathrm{RDW} \geq 14.8 \%$ (odds ratio, 17.549) and ferritin $<2,251.0 \mathrm{mg} / \mathrm{dL}$ (odds ratio, 32.414) independently suggested sepsis more than AOSD in patients initially presenting with fever requiring hospitalization. Conclusions: RDW might be a rapid and helpful marker for a differential diagnosis between AOSD from sepsis at an early phase.

Keywords: Red blood cell distribution width; Adult onset Still's disease; Sepsis; Differential diagnosis

\section{INTRODUCTION}

Adult onset Still's disease (AOSD) is a systemic inflammatory disorder characterized by persistent high fever, arthralgia/arthritis, and a typical rash among other diverse symptoms and signs [1]. Because no objective diagnostic markers for AOSD diagnosis have been es- tablished, the currently recommended classification criteria for AOSD are based on nonspecific and heterogeneous clinical and laboratory features after excluding other conditions such as infection and malignancy [1-3]. In actual clinical situations, it is quite difficult to discriminate AOSD and infectious diseases, especially sepsis, in patients presenting symptoms of systemic in- 
flammatory response syndrome as the initial manifestations $[1,4,5]$. Till date, several serological markers have been proposed for discriminating AOSD from sepsis $[4,6,7]$. However, as these markers may be increased in various conditions, complementary markers are still required [8].

The red blood cell distribution width (RDW) is an automatically measured value of the heterogeneity in the size of circulating red blood cells, and is usually used in distinguishing different types of anaemia [9]. Recently, it was reported that RDW associates with the pro-inflammatory cytokine levels, and that it can reflect the burden of inflammation and the prognosis in diverse diseases including sepsis [9-11]. Hence, in this study, we investigated whether RDW may significantly differ between AOSD and sepsis patients, and whether it can contribute to discriminating the two diseases.

\section{METHODS}

\section{Patients}

We reviewed the medical records of 30 AOSD patients, and 30 age- and gender-matched sepsis patients admitted and first diagnosed with AOSD or sepsis at Severance Hospital, Yonsei University College of Medicine, from January 2010 to March 2015. We included 21 of 30 AOSD patients, who fulfilled Yamaguchi's classification criteria for AOSD, and excluded nine patients with concomitantly confirmed connective tissue diseases or malignancies [1]. Sepsis was defined as a systemic inflammatory response syndrome accompanied by proven microbial infection [5]. We included 27 of 30 sepsis patients and excluded three patients in whom secondary infection due to underlying malignancies was strongly suspected. We also reviewed 30 age- and gender-matched healthy controls, who visited at the Yonsei University Medical Center for medical check-ups. This study was approved by the Institutional Review Board of Severance Hospital (4-2012-0092), who waived the need for patient written informed consent, as this was a retrospective study.

\section{Clinical data and laboratory results}

We obtained the items of clinical manifestations based on each disease entity as described in Table 1 [12]. Clin- ical signs were considered positive as described in our previous study [8]. We collected at least two laboratory results of the variables described in Table 2 within 24 hours after hospitalization and before the initiation of antibiotics, and calculated their mean values. RDW was measured using the Advia 2120 haematology analyzer (Siemens Healthcare Diagnostics, Deerfield, IL, USA).

\section{Statistical analysis}

All statistical analyses were conducted using the SPSS for Windows version 18.0 (SPSS Inc., Chicago, IL, USA). The frequencies of noncontinuous and continuous variables were expressed as the percentages and median values with interquartile range, respectively. Between-groups differences were assessed using the chi-square test and Fisher exact test for categorical results, and the Mann-Whitney $U$ test for continuous variables. The correlation between RDW and examined variables was evaluated using univariate Pearson correlation analysis. The optimal cutoff values of variables with statistical significance in the between-group analysis for predicting sepsis, including the white blood cell (WBC) count $\left(16,380.0 / \mathrm{mm}^{3}\right)$, RDW (14.8\%), and ferritin $(2,251.0 \mathrm{mg} / \mathrm{dL})$, were calculated by the area under the receiver operator characteristic curve according to the highest sum of the sensitivity and specificity. Odds ratios (ORs) for significant variables were obtained using multivariate logistic regression analysis. For all statistical evaluations, $p$ values $<0.05$ were considered to indicate statistical significance.

\section{RESULTS}

\section{Characteristics of patients and healthy controls}

The demographic and clinical features and the results of the culture studies of patients and healthy controls are described in Table 1. The median ages of patients with sepsis (nine men and 18 women), AOSD (five men and 16 women), and healthy controls (eight men and 22 women) were $47.0,44.0$, and 43.0 years, respectively. There were no significant differences in the median age or gender distribution among these three groups. In 27 patients with sepsis, bacteraemia was confirmed in 21 patients (77.8\%). In two of these 21 patients, other cultures studies disclosed no growth of micro-organism. Thirteen sepsis patients had pneumonia, eight had urinary tract 
Table 1. Characteristics of patients with sepsis and AOSD and healthy controls

\begin{tabular}{|c|c|c|c|}
\hline Variable & Patients with sepsis $(n=27)$ & Patients with AOSD $(n=21)$ & Healthy control $(n=30)$ \\
\hline \multicolumn{4}{|l|}{ Demographic variable } \\
\hline Age, yr & $47.0(42.0-50.0)$ & $44.0(33 \cdot 5-49 \cdot 5)$ & $43.0(37.8-49.3)$ \\
\hline Sex, male/female & $9 / 18$ & $5 / 16$ & $8 / 22$ \\
\hline \multicolumn{4}{|l|}{ Clinical feature } \\
\hline Fever & $27(100)$ & $21(100)$ & \\
\hline Arthralgia/arthritis & & $20(95.2)$ & \\
\hline Myalgia & & $17(81.0)$ & \\
\hline Typical skin rash & & $15(71.4)$ & \\
\hline Sore throat & & $12(57.1)$ & \\
\hline Lymphadenopathy & & $7(33 \cdot 3)$ & \\
\hline Pericarditis & & $7(33 \cdot 3)$ & \\
\hline Kidney involvement & & $5(23.8)$ & \\
\hline Hepatomegaly/splenomegaly & & $3(14 \cdot 3)$ & \\
\hline Pleuritis & & $3(14 \cdot 3)$ & \\
\hline Pneumonitis & & $2(9 \cdot 5)$ & \\
\hline Pulmonary arterial hypertension & & $1(4.8)$ & \\
\hline Systemic score ${ }^{\mathrm{a}}$ & & $6.48 \pm 2.04$ & \\
\hline \multicolumn{4}{|l|}{ Positivity of culture studies } \\
\hline Blood & $21(77.8)$ & & \\
\hline Sputum & $13(48.2)$ & & \\
\hline Urine & $8(29.6)$ & & \\
\hline Biliary drainage & $2(7 \cdot 4)$ & & \\
\hline Cerebrospinal fluid & $2(7 \cdot 4)$ & & \\
\hline Other infection & $2(7.4)$ & & \\
\hline
\end{tabular}

Values are presented as median (interquartile range), number (\%), or mean $\pm \mathrm{SD}$.

AOSD, adult onset Still's disease.

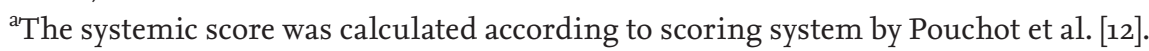

infection, two had hepatobiliary infection, and two had central nervous system infection. Arthralgia/arthritis (95.2\%) was the most frequently observed symptoms in febrile hospitalized AOSD patients, followed by myalgia (81.0\%), typical rash (71.4\%), and sore throat (57.1\%).

\section{Comparison of variables between patients with sep- sis and those with AOSD}

Sepsis patients showed significantly increased median WBC count and neutrophil proportion, compared to AOSD patients $\left(14,390.0 / \mathrm{mm}^{3} \mathrm{vs} .12,390.0 / \mathrm{mm}^{3}, p=0.010\right.$; $89.8 \%$ vs. $86.3 \%, p=0.023)$. The median RDW in sepsis patients was higher than that in AOSD patients $(15.0 \%$ vs. $13.3 \%, p=0.001$ ) (Table 2), and furthermore, the median RDWs in both patient-groups were significantly higher than that in healthy controls (Fig. 1). The haemoglobin and haematocrit values did not differ between sepsis and AOSD patients. In contrast, the median ferritin level in sepsis patients was lower than that in AOSD patients (544.0 vs. 3,756.6, $p=0.001$ ). There were no significant differences in other laboratory variables including erythrocyte sedimentation rate (ESR) and C-reactive protein (CRP) between the two groups (Table 2).

\section{Correlations between RDW and other variables}

When assessing the correlation between RDW and the clinically relevant variables in all sepsis and AOSD patients, we found that RDW was inversely correlated with haemoglobin and haematocrit $(r=-0.326$ and $r=-0.433$, both $p<0.05$ ). However, RDW showed no significant 
Table 2. Comparison laboratory variables between patients with sepsis and those with AOSD

\begin{tabular}{|c|c|c|c|}
\hline Variable & Patients with sepsis $(\mathrm{n}=27)$ & Patients with $\operatorname{AOSD}(\mathrm{n}=21)$ & $p$ value \\
\hline White blood cell, $/ \mathrm{mm}^{3}$ & $14,390.0(11,530.0-21,680.0)$ & $12,390.0(9,640.0-15,470.0)$ & 0.010 \\
\hline Neutrophil, \% & $89.8(84.6-94.7)$ & $86.3(83.7-89.6)$ & 0.023 \\
\hline Hemoglobin, g/dL & $10.6(9.7-12.7)$ & $11.8(10.0-13.1)$ & 0.264 \\
\hline Hematocrit, \% & $31.3(28.1-37.1)$ & $35.8(30.5-38.4)$ & 0.134 \\
\hline Red blood cell distribution width, \% & $15.0(13.2-16.7)$ & $13.3(13.1-14.2)$ & 0.001 \\
\hline Platelet, $/ \mathrm{mm}^{3}$ & $211,000.0(164,000.0-330,000.0)$ & $318,000.0(196,000.0-408,500.0)$ & 0.542 \\
\hline Ferritin, mg/dL & $544.0(289.0-1,143.0)$ & $3,756.6(784.6-11,480.7)$ & 0.001 \\
\hline Erythrocyte sedimentation rate, $\mathrm{mm} / \mathrm{hr}$ & $72.0(41.5-85.0)$ & $73.0(56.0-101.0)$ & 0.164 \\
\hline C-reactive protein, $\mathrm{mg} / \mathrm{dL}$ & $118.0(63.8-243.5)$ & $97.6(67.1-225.4)$ & 0.939 \\
\hline Blood urea nitrogen, mg/dL & $22.2(13.4-39.0)$ & $20.0(13.6-26.0)$ & 0.098 \\
\hline Creatinine, mg/dL & $1.0(0.7-1.3)$ & $0.9(0.7-1.0)$ & 0.138 \\
\hline Aspartate transaminase, IU/L & $34.5(23.5-91.8)$ & $46.0(27.0-50.0)$ & 0.085 \\
\hline Alanine transaminase, IU/L & $29.0(13.8-77.3)$ & $18.0(14 \cdot 5-35 \cdot 5)$ & 0.079 \\
\hline Lactate dehydrogenase, IU/L & $945.0(710.0-1,439.0)$ & $914.0(450.0-1,265 \cdot 5)$ & 0.108 \\
\hline Serum albumin, mg/dL & $3 \cdot 3(3.0-3.6)$ & $3.2(2.8-3.7)$ & 0.504 \\
\hline Total bilirubin, mg/dL & $1.3(0.5-2.4)$ & $0.5(0.3-0.9)$ & 0.066 \\
\hline Prothrombin time, INR & $1.1(1.0-1.3)$ & $1.1(1.0-1.2)$ & 0.578 \\
\hline Activated partial thromboplastin time, sec & $28.4(25.0-31.1)$ & $27.2(26.1-28.6)$ & 0.429 \\
\hline
\end{tabular}

Values are presented as median (interquartile range).

AOSD, adult onset Still's disease; INR, international normalized ratio.

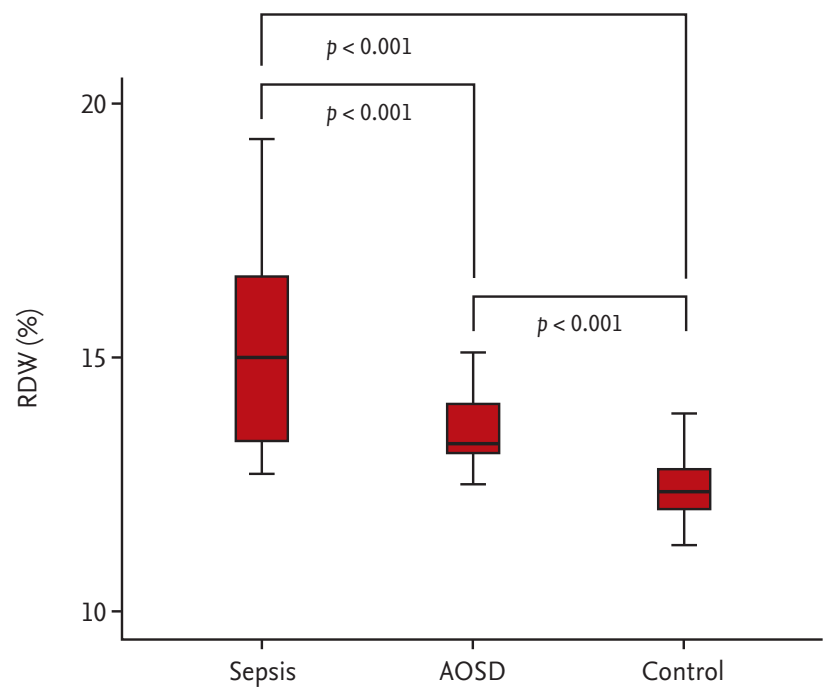

Figure 1. Comparison of the median red blood cell distribution width (RDW) among patient with sepsis and adult onset Still's disease (AOSD) and healthy controls. The median RDW in sepsis patients was higher than that in AOSD patients, and furthermore, the median RDW in both patientgroups was significantly higher than that in healthy controls (15.0\% for sepsis patients, $13.3 \%$ for AOSD patients, and $12.4 \%$ for healthy controls, all $p<0.001)$. correlation with patients' age, WBC count, ferritin, ESR, or CRP level (Table 3).

\section{Independent variables suggesting sepsis, compared to AOSD}

We identified the optimal cut-off value for predicting AOSD of variables that showed significant difference between the AOSD and sepsis groups, including the WBC count, RDW and ferritin using receiver operator characteristic curves. We selected a WBC count of $16,380 / \mathrm{mm}^{3}$ (sensitivity, 48.1\%; specificity, $85.7 \%$ ), a ferritin level of 2,251 mg/dL (sensitivity, 61.9\%; specificity, 96.3\%), and a RDW of $14.8 \%$ (sensitivity, 51.9\%; specificity, 95.2\%) as the optimal cut-off values for discriminating sepsis from AOSD.

All patients were classified into two groups according to these cut-offs, resulting in significant differences in the proportions of WBC counts $\geq 16,380 / \mathrm{mm}^{3}$, RDW $\geq$ $14.8 \%$, and ferritin $<2,251 \mathrm{mg} / \mathrm{dL}$ between patients with AOSD and sepsis in the univariate logistic regression. Further, in the multivariate analysis, RDW $\geq 14.8 \%$ (OR, 17.549; 95\% confidence interval [CI], 1.123 to $274.166 ; p=$ 
Table 3. Correlation between red blood cell distribution width and variable

\begin{tabular}{lcc}
\hline Variable & $\begin{array}{c}\text { Correlation } \\
\text { coefficient }(r)\end{array}$ & p value \\
\hline Age, yr & 0.126 & 0.394 \\
Hemoglobin, g/dL & -0.326 & 0.024 \\
Hematocrit, \% & -0.433 & 0.002 \\
White blood cell, /mm $\mathrm{mm}^{3}$ & 0.270 & 0.064 \\
Ferritin, mg/dL & -0.187 & 0.204 \\
Erythrocyte sedimentation & -0.093 & 0.543 \\
rate, mm/hr & & \\
C-reactive protein, mg/dL & 0.015 & 0.917 \\
\hline
\end{tabular}

0.041) and ferritin < 2,251.0 mg/dL (OR, 32.414; 95\% CI, 2.854 to $368.082 ; p=0.005)$ independently and meaningfully suggested sepsis more frequently than AOSD in patients initially presenting with fever requiring hospitalization (Table 4).

\section{DISCUSSION}

In this study, we found that the mean RDW in AOSD and sepsis patients were significantly higher than that in healthy controls, and particularly, RDW in AOSD patients remarkably lower than that in sepsis patients (mean value 13.3 vs. 15.0, $p=0.001$ ) along with $\mathrm{WBC}$ count and ferritin level. When all patients were classified into two groups according to the optimal cut-off of RDW (14.8\%), the relative risk for sepsis in patients having RDW more than $14.8 \%$ than those having RDW below this cut-off was increased by 21.5 -fold in the univariate analysis. Furthermore, in multivariate analysis, RDW $\geq 14.8 \%$ (OR, 17.549) and ferritin < 2,251.0 mg/dL (OR, 32.414) significantly predict sepsis in patients presenting similar clinical and laboratory features to AOSD. Therefore, although the discriminable potential of RDW did not surpass that of ferritin level, considering the statistical significance, RDW may represent a complementary marker to establish the differential diagnosis between AOSD and sepsis in febrile patients presenting indistinguishable or similar clinical and laboratory features within 24 hours after hospitalization.

Among several confounding factors, anaemia is a leading factor affecting RDW [9]. In this study, RDW also showed significant correlation with both haemoglobin and haematocrit. However, there were no significant differences in those variables between AOSD and sepsis patients. This result suggests that RDW in the very early phase of AOSD or sepsis might reflect the burden of inflammation more than the underlying or proceeding anaemia status. The mechanism of elevated RDW in inflammatory conditions remains unclear. Proinflammatory cytokines might stimulate the proliferation of erythroid progenitor cells, and suppress the maturation of red blood cells, resulting in the release of immature red blood cell into the peripheral blood stream $[4,13,14]$. Meanwhile, ESR and CRP, the most widely used acute phase reactants, neither differed between AOSD and sepsis patients, nor correlated with RDW, unlike in previous studies [15]. Based on these results, we carefully speculate that RDW might reflect precise changes in narrow spectrum of inflammation, compared to ESR and CRP, which show a wide range of alterations in AOSD and sepsis patients within 24 hours after hospitalization. Furthermore, RDW is included in the complete blood count test, which is routinely per-

Table 4. Independent variables suggesting sepsis compared to AOSD

\begin{tabular}{|c|c|c|c|c|c|c|}
\hline \multirow{2}{*}{ Variable } & \multicolumn{3}{|c|}{ Univariate } & \multicolumn{3}{|c|}{ Multivariate } \\
\hline & OR & $95 \% \mathrm{CI}$ & $p$ value & OR & $95 \% \mathrm{CI}$ & $p$ value \\
\hline White blood cell, $\geq 16,380.0 / \mathrm{mm}^{3}$ & $5 \cdot 571$ & $1.325-23.435$ & 0.019 & $3 \cdot 717$ & $0.500-27.661$ & 0.200 \\
\hline White blood cell, $<16,380.0 / \mathrm{mm}^{3}$ & Reference & & & Reference & & \\
\hline Red blood cell distribution width, $\geq 14.8 \%$ & 21.538 & $2.520-184.085$ & 0.005 & $17 \cdot 549$ & $1.123-274.166$ & 0.041 \\
\hline Red blood cell distribution width, <14.8\% & Reference & & & Reference & & \\
\hline Ferritin, $<2,251.0 \mathrm{mg} / \mathrm{dL}$ & 42.250 & $4.762-374.828$ & 0.001 & 32.414 & $2.854-368.082$ & 0.005 \\
\hline Ferritin, $\geq 2,251.0 \mathrm{mg} / \mathrm{dL}$ & Reference & & & Reference & & \\
\hline
\end{tabular}

AOSD, adult onset Still's disease; OR, odds ratio; CI, confidence interval. 
formed, without additional costs or tests, when patients with fever are admitted to a hospital. Given that there are only a few objective laboratory results to help physicians to discriminate AOSD and sepsis in their early phase, we suggest that RDW might be useful in making the differential diagnosis of AOSD from sepsis as a rapid and cost-effective supplementary tool to ferritin, although RDW should not be used as an independent predictive value for the differential diagnosis between the two diseases.

This study has several limitations that need to be addressed, including the small number of AOSD patients due to its low incidence, and the fact that no analysis of proinflammatory cytokines could be performed because of the retrospective design of the study. To overcome these issues, future studies including larger numbers of AOSD and sepsis patients and that measure the serum levels of inflammatory cytokines serially should be performed and might reveal a dynamic role of RDW in the differential diagnosis of AOSD from sepsis in the early phase.

In conclusion, RDW might be a rapid and helpful marker for the differential diagnosis between AOSD and sepsis at the early phase when their disease patterns are indistinguishable, supplementary to ferritin.

\section{KEY MESSAGE}

1. It is difficult to discriminate adult onset Still's disease (AOSD) and sepsis, in patients presenting symptoms of systemic inflammatory response syndrome as initial manifestations.

2. In this study, red blood cell distribution width $(\mathrm{RDW}) \geq 14.8 \%$ independently and meaningfully suggested sepsis more than AOSD in patients initially presenting with fever requiring hospitalization, comparable to ferritin.

3. RDW might be a rapid and helpful marker for a differential diagnosis between AOSD and sepsis at an early phase when their disease patterns are indistinguishable, supplementary to ferritin.

\section{Conflict of interest}

No potential conflict of interest relevant to this article was reported.

\section{Acknowledgments}

This study was supported by a grant from the Basic Science Research Program through the National Research Foundation of Korea (NRF) funded by the Ministry of Education, Science and Technology (2012R1A1A1002580) and the Korea Health Technology R\&D Project through the Korea Health Industry Development Institute, funded by the Ministry of Health and Welfare, Republic of Korea (HI14C1324).

\section{REFERENCES}

1. Yamaguchi M, Ohta A, Tsunematsu T, et al. Preliminary criteria for classification of adult Still's disease. J Rheumatol 1992;19:424-430.

2. Fautrel B, Zing E, Golmard JL, et al. Proposal for a new set of classification criteria for adult-onset still disease. Medicine (Baltimore) 2002;81:194-200.

3. Cush JJ. Adult-onset Still's disease. Bull Rheum Dis 2000;49:1-4.

4. Priori R, Colafrancesco S, Alessandri C, et al. Interleukin 18: a biomarker for differential diagnosis between adultonset Still's disease and sepsis. J Rheumatol 2014;41:11181123 .

5. Klein Klouwenberg PM, Ong DS, Bonten MJ, Cremer OL. Classification of sepsis, severe sepsis and septic shock: the impact of minor variations in data capture and definition of SIRS criteria. Intensive Care Med 2012;38:811819.

6. Van Reeth C, Le Moel G, Lasne Y, et al. Serum ferritin and isoferritins are tools for diagnosis of active adult Still's disease. J Rheumatol 1994;21:890-895.

7. Park HJ, Ha YJ, Pyo JY, Park YB, Lee SK, Lee SW. Delta neutrophil index as an early marker for differential diagnosis of adult-onset Still's disease and sepsis. Yonsei Med J 2014;55:753-759.

8. Lee SW, Park YB, Song JS, Lee SK. The mid-range of the adjusted level of ferritin can predict the chronic course in patients with adult onset Still's disease. J Rheumatol 2009;36:156-162.

9. Evans TC, Jehle D. The red blood cell distribution width. J Emerg Med 1991;9 Suppl 1:71-74.

10. Lorente L, Martin MM, Abreu-Gonzalez P, et al. Red blood cell distribution width during the first week is associated with severity and mortality in septic patients. 
PLoS One 2014;9:e105436.

11. Dabbah S, Hammerman H, Markiewicz W, Aronson D. Relation between red cell distribution width and clinical outcomes after acute myocardial infarction. Am J Cardiol 2010;105:312-317.

12. Pouchot J, Sampalis JS, Beaudet F, et al. Adult Still's disease: manifestations, disease course, and outcome in 62 patients. Medicine (Baltimore) 1991;70:118-136.

13. Scharte M, Fink MP. Red blood cell physiology in critical illness. Crit Care Med 2003;31(12 Suppl):S651-S657.

14. Ghaffari S. Oxidative stress in the regulation of normal and neoplastic hematopoiesis. Antioxid Redox Signal 2008;10:1923-1940.

15. Lippi G, Targher G, Montagnana M, Salvagno GL, Zoppini G, Guidi GC. Relation between red blood cell distribution width and inflammatory biomarkers in a large cohort of unselected outpatients. Arch Pathol Lab Med 2009;133:628-632. 